\title{
Designer probiotics: a potential therapeutic for Clostridium difficile?
}

The continuing demographic shift towards a more elderly society, coupled with an ever increasing dependence on conventional therapeutics and an alarming escalation in antibiotic resistance, has facilitated the emergence of a new faction of bacterial adversaries, the 'superbugs', the aetiological agents of nosocomial infections. One of the most notorious of this emerging group is Clostridium difficile, the most common identifiable cause of bacteria-associated diarrhoea in the United States and the major cause of gastroenteritis in nursing homes and health-care facilities for the elderly (Crogan \& Evans, 2007).

In addition to hospitalization, the most significant predisposing factors for $C$. difficile infection include advanced age ( $>65$ years) and antibiotic therapy. The most common antibiotic inducing agents implicated to date include the broadspectrum cephalosporins, while the only remaining effective therapeutic agents are metronidazole and vancomycin (McFarland, 2005). Despite being the most effective antibiotic, vancomycin use carries with it the added risk of secondary colonization by vancomycin-resistant enterococci and/or the spread of vancomycin resistance to other potentially dangerous hospital 'superbugs' such as meticillin-resistant Staphylococcus aureus.

Against this backdrop, the last decade has seen the emergence of a new epidemic of C. difficile-associated disease (CDAD) (Kuijper et al., 2007). Linked to the hypervirulent ribotype 027 , this epidemic is characterized by increased frequency and severity of enteric disease and is significantly more recalcitrant to standard antibiotic therapy. Faced with this epidemic, clinicians and researchers alike are now struggling to find viable therapeutic alternatives (McFarland, 2005). One such alternative involves the use of probiotics; these are defined as live microorganisms, which when consumed in adequate amounts, confer a health benefit on the host'. Probiotic therapy has become the focus of considerable research efforts in recent times (Sleator \& Hill, 2007a). Indeed, numerous clinical studies have attributed a myriad of impressive health-promoting effects to probiotics, including effective treatment of certain digestive and metabolic disorders as well as antagonistic activities against a variety of microbial pathogens. Hickson et al. (2007) recently reported that consumption of a commercially available probiotic drink can reduce the incidence of CDAD in a hospital setting and has the potential to decrease health-care costs, morbidity and mortality if used routinely in patients aged over 50 .

While the exact mechanisms by which probiotic bacteria inhibit pathogens such as C. difficile are as yet poorly understood, some advances have nevertheless been made in our understanding of probiotic function. Recent work in our laboratory, for example, revealed that the therapeutic potential of the probiotic strain Lactobacillus salivarius is due, at least in part, to its ability to produce a potent two-peptide bacteriocin, Abp118 (Corr et al., 2007). Furthermore, Rea et al. (2007) recently showed significant anti-C. difficile potential for yet another bacteriocin, the two-component lantibiotic lacticin 3147, produced by Lactococcus lactis. Significantly, and in contrast to conventional broad-spectrum antibiotics, lacticin 3147 completely eliminates $10^{6}$ c.f.u. C. difficile $\mathrm{ml}^{-1}$ within $30 \mathrm{~min}$ (at concentrations as low as $18 \mu \mathrm{g} \mathrm{ml}^{-1}$ ) without dramatically impacting on the normal resident microflora. While this work involved in vitro studies in model faecal environments, in vivo sensitivity of the bacteriocin to gastric acidity creates a technological/delivery hurdle which will have to be overcome if this bacteriocin is to achieve its potential as an effective oral therapeutic (Gardiner et al. 2007).

A novel solution to the loss of activity associated with direct ingestion of the bacteriocin is to clone and express the genes for bacteriocin production (and immunity) into an appropriate bacterial carrier, such as Lactobacillus salivarius. This approach circumvents in vivo degradation of the bacteriocin during gastric transit and facilitates continued bacteriocin production at the site of infection (the sigmoid colon), while at the same time dramatically improving the clinical efficacy of the probiotic (Fig. 1a). Such 'designer probiotics' may be further manipulated, using a patho-biotechnologybased approach (Sleator \& Hill, 2006, 2007b), to improve growth and survival potential both ex vivo (Sheehan et al., 2006) and in vivo (Sheehan et al., 2007).

In addition to modulating their antimicrobial spectrum and improving their physiological stress tolerance, recent studies have led to the development of probiotic strains engineered to express receptor mimics on their surface. These surface proteins specifically target enteric infections by blocking crucial ligandreceptor interactions between the pathogen and host cell (Paton et al., 2006; Fig. 1b). Blocking this adherence reduces infection, while toxin neutralization ameliorates symptoms until the pathogen is eventually overcome by the immune system, as a result of bacteriocin production, or combined antibiotic therapy. Given that a significant feature of the C. difficile epidemic strain 027 is increased production of toxin A and B (Warny et al., 2005), the ability to neutralize or 'mop up' these toxins would result in a significantly improved prognosis (Fig. 1c). In support of this proposal, Paton et al. (2000) described the construction of a probiotic Escherichia coli strain expressing a chimeric lipopolysaccharide terminating in a shiga toxin (Stx) receptor, $1 \mathrm{mg}$ dry weight of which can neutralize $>100 \mu \mathrm{g}$ Stx1 and Stx2. Designer probiotics with receptor blocking potential against enterotoxigenic E. coli toxin LT and cholera toxin (Ctx) have also been described (Paton et al., 2006). 


\section{Gut Lumen}

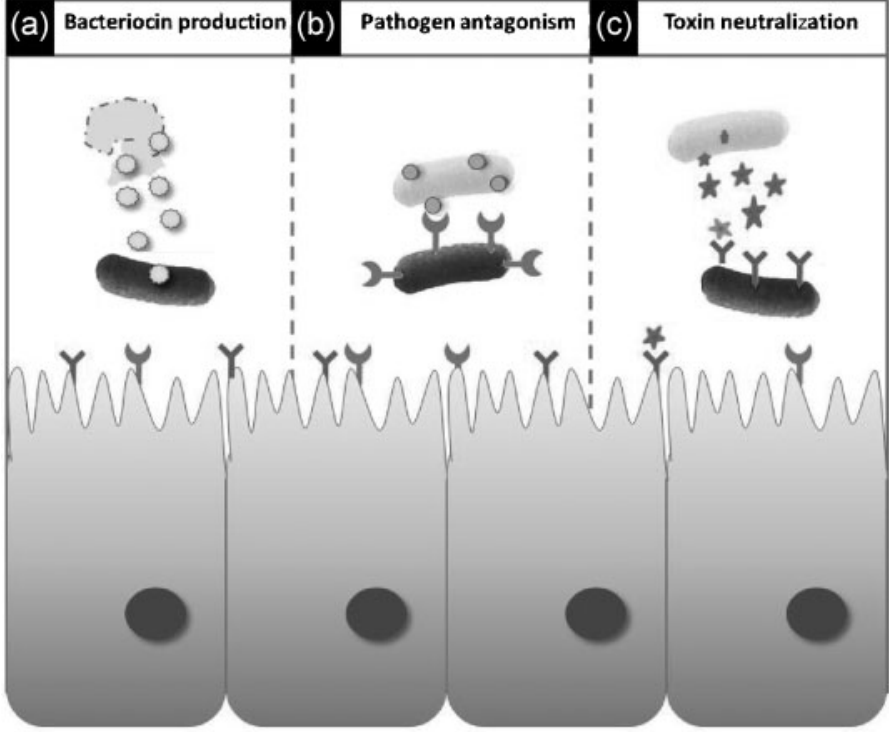

Basolateral surface of the intestinal epithelial cells

Fig. 1. Overview of the anti-C. difficile potential of designer probiotics. Bacteriocin produced by the probiotic (dark shading) can lyse invading C. difficile cells (light shading) (a), while heterologously expressed receptor mimics on the surface of probiotic cells can antagonize pathogen adherence to the host (b) and neutralize toxin production (c).

In conclusion then, while conventional medical research continues in its attempts to develop effective therapeutic and prophylactic compounds against $C$. difficile, their application is often complicated by in vivo sensitivity and rising production costs. 'Designer probiotics' on the other hand provide an effective means of circumventing the short half-life and fragility of conventional therapeutics, providing a cost-effective alternative which will ultimately contribute to health and social gain (Sleator \& Hill, 2007c). Indeed, McFarland (2005) in her seminal review on alternative approaches to the control of $C$. difficile proposed that effective treatment of CDAD needs to 'reduce the burden of $C$. difficile and its toxins in the intestine, restore the normal colonic microflora and assist the host's immune system'. Designer probiotics satisfy all three of these requirements, thus making them an ideal alternative treatment for $C$. difficile. However, while consumer acceptance of genetically engineered micro-organisms still remains a significant challenge, this obstacle should eventually be overcome by the application of rigorous scientific controls, such as adequate biological containment, and proper riskbenefit analysis of the potential advantages of such a strategy.

\section{Acknowledgements}

R.D.S. is a Health Research Board (HRB) Principal Investigator. The authors wish to acknowledge the continued financial assistance of the HRB and Alimentary Pharmabiotic Centre (APC) through funding by Science Foundation Ireland (SFI). R.D.S. was the recipient of a Marie Curie travel grant to attend the Second International Clostridium difficile Symposium held in Maribor, Slovenia, 6-9 June 2007.

\section{Roy D. Sleator and Colin Hill}

Alimentary Pharmabiotic Centre, University College Cork, College Road, Cork, Ireland

Correspondence: Roy D. Sleator (r.sleator@ucc.ie)

Corr, S. C., Li, Y., Riedel, C. U., O'Toole, P. W., Hill, C. \& Gahan, C. G. (2007). Bacteriocin production as a mechanism for the antiinfective activity of Lactobacillus salivarius UCC118. Proc Natl Acad Sci U S A 104, 7617-7621.
Crogan, N. L. \& Evans, B. C. (2007). Clostridium difficile: an emerging epidemic in nursing homes. Geriatr Nurs 28, 161-164.

Gardiner, G. E., Rea, M. C., O' Riordan, B., O' Connor, P., Morgan, S. M., Lawlor, P. G., Lynch, P. B., Cronin, M., Ross, R. P. \& Hill, C. (2007). Fate of the two-component lantibiotic lacticin 3147 in the gastrointestinal tract. Appl Environ Microbiol 73, 7103-7109.

Hickson, M., D'Souza, A. L., Muthu, N., Rogers, T. R., Want, S., Rajkumar, C. \& Bulpitt, C. J. (2007). Use of probiotic Lactobacillus preparation to prevent diarrhoea associated with antibiotics: randomised double blind placebo controlled trial. BMJ 335, 80 .

Kuijper, E. J., van Dissel, J. T. \& Wilcox, M. H. (2007). Clostridium difficile: changing epidemiology and new treatment options. Curr Opin Infect Dis 20, 376-383.

McFarland, L. V. (2005). Alternative treatments for Clostridium difficile disease: what really works? J Med Microbiol 54, 101-111.

Paton, A. W., Morona, R. \& Paton, J. C. (2000). A new biological agent for treatment of Shiga toxigenic Escherichia coli infections and dysentery in humans. Nat Med 6, 265-270.

Paton, A. W., Morona, R. \& Paton, J. C. (2006). Designer probiotics for prevention of enteric infections. Nat Rev Microbiol 4, 193-200.

Rea, M. C., Clayton, E., O'Connor, P. M. Shanahan, F., Kiely, B., Ross, R. P. \& Hill, C. (2007). Antimicrobial activity of lacticin 3147 against clinical Clostridium difficile strains. J Med Microbiol 56, 940-946.

Sheehan, V. M., Sleator, R. D., Fitzgerald, G. F. \& Hill, C. (2006). Heterologous expression of BetL, a betaine uptake system, enhances the stress tolerance of Lactobacillus salivarius UCC118. Appl Environ Microbiol 72, 2170-2177.

Sheehan, V. M., Sleator, R. D., Hill, C. \& Fitzgerald, G. F. (2007). Improving gastric transit, gastrointestinal persistence and therapeutic efficacy of the probiotic strain Bifidobacterium breve UCC2003. Microbiology 153, 3563-3571.

Sleator, R. D. \& Hill, C. (2006)

Patho-biotechnology: using bad bugs to do good things. Curr Opin Biotechnol 17, 211-216.

Sleator, R. D. \& Hill, C. (2007a). Improving probiotic function using a patho-biotechnology approach. Gene Ther Mol Biol 11, 269-274.

Sleator, R. D. \& Hill, C. (2007b).

Patho-biotechnology; using bad bugs to make good bugs better. Sci Prog 90, 1-14.

Sleator, R. D. \& Hill, C. (2007c). Probiotics as therapeutics for the developing world. J Infect Developing Countries 1, 7-12.

Warny, M., Pepin, J., Fang, A., Killgore, G., Thompson, A., Brazier, J., Frost, E. \& McDonald, L. C. (2005). Toxin production by an emerging strain of Clostridium difficile associated with outbreaks of severe disease in North America and Europe. Lancet 366, 1079-1084. 